\title{
Targeting Adenoviral Entry to Enhance Oncolytic Antitumor Response
}

\author{
Hidde J. Haisma* and Lieke Geerts
}

Pharmaceutical Gene Modulation, Groningen Research Institute of Pharmacy, Groningen University, A. Deusinglaan 1, 9713 AV Groningen, Netherland

\begin{abstract}
Conditionally replicative adenoviruses represent an innovative group of anticancer agents designed to destroy these cells by replication and lysis. A major problem associated with of the use of adenoviral vectors in gene therapy is its high liver uptake and lack of tumor selectivity upon systemic administration. To improve the efficacy of CRAds as anticancer agents, their infection efficiency on CAR-deficient tumor cells could be enhanced their by redirecting viral entry via a CAR-independent pathway. To redirect the entry pathway of adenoviruses and enhance their infectivity and specificity, two general strategies are being used. In the first strategy, the adenovirus genome is changed to alter the binding specificity of the viral capsid. In the second strategy, a two-component targeted adenovirus is created by binding of proteins with specific affinity for cancer cells onto the viral capsid. Despite effective targeting and tumor eradication in vitro and in mouse models, the results from systemic administration of targeted CrAds is limited. In addition, clinical effects of CrAds are disappointing up till now. Therefore, combination therapies in which targeted CrAds are combined with other types of therapy are being investigated.
\end{abstract}

Keywords: Conditionally replicative adenoviruses, targeting, peptides, bispecific single-chain antibodies.

\section{INTRODUCTION}

\section{Cancer Gene Therapy}

Gene therapy involves the delivery of genes to specific cells of interest in order to treat a disease. In this manner, gene therapy can theoretically be used to deliver toxin or corrective genes to tumor cells specifically, which would be an innovative and promising treatment strategy for cancer. This is limited in practice however, due to a low efficiency and specificity of gene delivery to target cells. Improved delivery vehicles are therefore required for a specific gene delivery and expression in target cells, without harming healthy cells.

Delivery by viral vectors is the most common systemic delivery strategy currently being investigated in gene therapy. Viral vectors are used in almost $70 \%$ of clinical trials in gene therapy, $24.7 \%$ being adenoviral vectors [1]. Advantages of the adenovirus are its high expression of transgenes, its large DNA payload capacity, its high stability and low pathogenicity in vivo and its ability to infect quiescent as well as dividing cells. Conditionally replicative adenoviruses [CRAds] represent a novel class of anticancer agents designed to selectively replicate in tumor cells and to destroy these cells by inducing lysis [2, 3].

\section{ADENOVIRAL CELL ENTRY BIOLOGY}

The basic adenoviral structure is an icosahedron capsid formed by hexon proteins. A penton assembly is attached to

\footnotetext{
*Address correspondence to this author at the Pharmaceutical Gene Modulation, Groningen Research Institute of Pharmacy, Groningen University, A. Deusinglaan 1, 9713 AV Groningen, Netherland; Tel: +31 50 3637866; Fax: +31 50 3637953; E-mail: h.j.haisma@rug.nl
}

the vertices, formed by the penton base and fiber proteins protruding from it [4].

Human Ad serotypes of subgroup B [serotypes 3, 7, 16, $21,50,11,14,34,35$ ] use CD46 as a primary attachment receptor [5]. CD46 is a member of a family of proteins that regulate complement activation and is expressed on all human cells with the exception of erythrocytes [6].

Binding of adenoviruses serotypes of subgroup A, D, E and $\mathrm{F}$ is mediated by the tethering receptor CAR [coxsackie and adenovirus receptor] on the cell surface, comprising the first step in cell entry of the virus $[5,7,8]$. The immunoglobulin-like D1 domain of this CAR receptor is able to bind to the carboxyterminal knob domain of serotype 5 fiber [9], following cell-surface $\alpha v \beta 3$ and $\alpha v \beta 5$ integrin mediated virus internalization through the arginine-glycine aspartic acid [RGD] sequence of the adenoviral penton base protein [10]. The viral particle is internalized into a clathrin-coated endosome, from which it can be released into the cytoplasm through acidification of the endosome [11]. After release the particle is being translocated to the nucleus, following replication of the virus.

A major drawback of the use of adenoviral vectors in gene therapy is its high liver tropism and lack of tumor tropism upon systemic delivery. Over $90 \%$ of intravenously administered adenovirus is rapidly taken up by the liver Kupffer cells through the scavenger receptor [12], giving rise to hepatotoxicity, a reduction in the viral transduction of target cells and thus decreased therapeutic efficiency.

Unfortunately, primary human cancers express low levels of CAR and are refractory to adenovirus infection [13-18]. In particular, high-grade undifferentiated tumors exhibited decreased CAR expression $[19,20]$. Furthermore, primary 
tumors revealed heterogeneous CAR expression, sometimes with a focal distribution pattern $[14,20]$. Thus, lack of CAR expression in primary tumors or tumor regions may hinder CRAd-based therapies. Therefore, abolishment of CAR mediated adenoviral cell entry and tumor retargeting has received much attention in studies focusing on improvement of adenoviral targeting specificity.

\section{CONDITIONALLY REPLICATIVE ADENOVIRUSES [CRAds]}

Conditionally replicative adenoviruses [CRAds] represent a novel class of anticancer agents designed to selectively replicate in tumor cells and to destroy these cells by inducing lysis [2, 3]. The first CRAd developed, is known as ONYX015 or d11520. It carries a deletion in the E1B-55kDa coding region that introduces selectivity for tumors with dysfunctional p53 [21]. The normal function of the E1B $55 \mathrm{kDa}$ protein is to bind to and inactivate p53 protein in infected cells. Because Onyx-015 lacks this protein, it was originally thought to replicate in a p53-dependent manner. Later it was shown that tumor selectivity of this CrAd was because of an altered nuclear RNA export pathways [22]. This approach has potential advantages over the traditional therapies of chemotherapy and radiotherapy because of the possibility of targeting tumors at a molecular level while leaving normal tissue relatively unaffected [23].

Promising preclinical results led to rapid translation to clinical trials for head and neck cancer [24, 25], pancreas carcinomas [26], and malignant glioma [27]. ONYX-015 was infused intravenously in patients with advanced carcinoma metastatic to the lung. No dose-limiting toxicity was identified [23, 25]. Clinical efficacy of this virus however was low [28, 29]. In 2006 the first intratumoral administrated oncolytic adenovirus was clinically approved to treat headand-neck cancer patients in a combination with chemotherapeutic treatment [30].

Other types of CRAd include the Ad-delta-24 or d1922947 which carries a partial deletion in the CR2 domain of the adenoviral E1A gene that abrogates the binding of E1A to $\mathrm{pRb}$ for the treatment of glioma [3, 31-33]. Epstein-Barr virus specific replication in nasopharyngeal carcinoma [34], human papillomavirus in anogenital cancers [35], tyrosinase to target melanoma [36, 37], CXCR4 to target ovarian cancer [38] and PSA for prostate cancer [39] for a review see [40, 41] and this issue.

\section{TARGETING STRATEGIES FOR ONCOLYTIC ADENOVIRUS}

A rational approach to improve the efficacy of CRAds as anticancer agents could be to enhance their infection efficiency on CAR-deficient tumor cells by redirecting viral entry via a CAR-independent pathway. This should augment the infection efficiency during replication and lateral spread in a CAR-deficient or heterogeneous tumor. Currently, two general strategies are being considered to redirect the entry pathway of adenoviruses in order to enhance their infectivity and specificity. In the first strategy, the adenovirus genome is modified to alter the binding specificity of the viral capsid [42]. In the second strategy, a two-component targeted ade- novirus is constructed by coupling proteins with specific binding affinity onto the viral capsid [14, 16, 18, 43-48].

\section{PSEUDOTYPED ADENOVIRUSES}

In the context of CRAds, the modification of the adenovirus genome to alter the binding specificity of the viral capsid appears most appropriate because the targeting specificity is made inherent to the viral genome and thus maintained upon replication. Many human Ad serotypes of subgroup A, $\mathrm{D}, \mathrm{E}$ and $\mathrm{F}$ recognize CAR whereas CD46 was identified as a cellular receptor for the majority of subgroup B [serotypes $3,7,16,21,50,11,14,34,35$ ] Ads [5]. Thus exchanging the capsid proteins from one serotype with another should confer a different cell specificity.

The utility of this strategy was confirmed by oncolytic replication of an adenovirus with a chimeric Ad3/Ad5 fiber protein to target infection via the putative $\mathrm{Ad} 3$ receptor in renal cell carcinoma models resistant to Ad5 infection [49] and head and neck cancer. $[26,50,51]$ The cyclooxygenase2 [Cox-2L] promoter was found to be the most advantageous in pancreatic cancer cell lines. An Ad5/Ad3 CRAd with replication of the virus controlled by the Cox-2L promoter was found to safely exhibit replication within a tumor in this model and was found to suppress tumor growth after systemic delivery [52].

Stoff-Khalili MA et al. [53] constructed an oncolytic adenovirus using the human CXCR4 gene promoter in an 5/3 fiber-modified CRAd. The oncolytic activity of this virus was studied in breast cancer cell lines, primary breast cancer and human liver tissue slices from patients, and in a xenograft breast cancer mouse model. The pseudotyped CRAd agent showed improved replication and killing in breast cancer cells in vitro and in vivo with a remarkable specificity profile that was strongly attenuated in non-breast cancer cells, as well as in normal human breast and liver tissues.

Guse K [54] took this approach one step further and constructed Ad5/3-9HIF-Delta24-VEGFR-1-Ig, an oncolytic pseudotyped adenovirus secreting soluble VEGF receptor to block angiogenesis. In an intraperitoneally disseminated kidney cancer model, significantly enhanced survival was observed with the Ad5/3-9HIF-Delta24-VEGFR-1-Ig when compared with control viruses.

Hoffmann D et al. [55] used Ad5/35 fiber chimeric adenovirusses with vector binding redirected to the Ad35 receptor and replication under the control of the GFAP/Ki67 or E2F-1/COX-2 promoters. The native Ad5 was compared to the chimeric Ad5/35 fiber for their antineoplastic activity in a subcutaneous and intracranial glioblastoma xenograft model. Animals treated with the Ad5/35-based vectors showed significantly smaller tumors and longer survival than those treated with the homologous Ad5 vectors.

\section{INCORPORATION OF TARGETING PEPTIDES}

Although useful to demonstrate the benefit of targeting, application of pseudotyped adenoviruses is limited by the targeting repertoire of naturally occurring adenovirus serotypes. As an alternative, CRAd tropism was expanded 
through incorporation of defined targeting peptides in the Ad5 fiber knob.

Insertion of the RGD [Arg-Gly-Asp] sequence, known to interact with $\mathrm{v}$ integrins, into the Ad fiber knob improved oncolytic potency on cancer cells in vitro and in vivo [56, 57]. It was demonstrated that combining this strategy with the Ad-delta-24 CRAd led to strong oncolytic effects in lung adenocarcinoma, prostate cancer, glioma and ovarian cancer cells [58-63]. To further improve tumor selectivity Cascallo et al. [64] combined the E2F promoter with the the $\mathrm{Rb}$ selectivity of delta 24 deletion in the E1 gene of the adenovirus in an RGD targeted virus. A single intravenous administration in three preclinical models in mice showed significant tumor growth inhibition [65].

Ranki et al. [66] generated a novel p16/retinoblastoma pathway-dependent CRAd, Ad5.pK7-Delta24, with a polylysine motif [Lys 7] in the fiber C-terminus, enabling CAR-independent binding to heparan sulfate proteoglycans [HSPG]. Ad5.pK7-Delta24 mediated effective oncolysis of breast cancer cell lines in vitro. A therapeutic benefit was seen following both intratumoral and intravenous delivery in an orthotopic model of advanced hormone refractory breast cancer. Piao Y et al. [67] described a Delta-24 adenovirus which was retargeted through the abrogation of CAR binding [Y477A mutation in the adenoviral fiber protein] and insertion of an epidermal growth factor receptor[EGFR]-specific binding peptide in the HI loop of the fiber protein. Treatment with this CrAd prolonged the survival of animals with intracranial xenografts derived from glioma cells. Similarly, a survivin driven oncolytic adenovirus targeted to heparan sulfate, was shown to be superior to Ad targeted to CD46 or RGD in glioma [68].

Nishimoto $\mathrm{T}$ et al. [69] evaluated a targeted adenovirus derived from a random peptide library displayed on an adenoviral fiber knob. In this approach one may overcome the limitation that many cell type-specific ligands are not compatible with adenovirus fiber assembly. Replicationcompetent adenovirus displaying selected peptides showed higher oncolytic potency in several pancreatic cancer cell lines compared with the untargeted adenovirus. Intratumoral injection of the peptide-targeted CrAd significantly suppressed the growth of subcutaneous tumors.

Thus, the potential of peptide-targeted CRAds for the treatment of cancer is clearly established. A major obstacle for wider application of fiber-modified CRAds with other recognition specificities is, however, that the adenovirus fiber appears to impose not yet fully understood structural demands on the incorporated ligand [70-72]. In general, only relatively small targeting ligands have been successfully rescued into native Ad fiber; presumably, incorporation of larger motifs destabilizes fiber structure and prevents trimerization. As an alternative, another Ad capsid protein, pIX, can be used to present large fusion proteins on the outside of the Ad capsid [73]. For example, Meulenbroek et al. [74] generated a pIX-green fluorescent protein [GFP] fusion protein and showed that it is efficiently incorporated into the Ad capsid. pIX . In addition, the adenovirus fiber shaft or hexon has been modified to contain an RGD peptide motiv
$[75,76]$. In order to allow the incorporation of larger peptides, Belousova et al. [70] replaced the Ad fiber with the trimerization domain of the phage T4 fibritin, and showed that the fibritin could be fused to the human CD40 ligand. CD40 belongs to the tumor necrosis factor [TNF] receptor superfamily and is highly expressed on myelomas, dendritic cells and tumor cells cells.

Thus larger binding ligands may be incorporated into the Adenovirus capsid by incorporation into either pIX or a modified fiber protein, however, thusfar these have not been used in the context of replication competent Ads.

\section{ADAPTER TARGETED CRAd}

The repertoire of genetically targeted CRAds could potentially be expanded tremendously if more complex, highaffinity ligands such as antibodies could be used. However, such molecules have so far not been successfully incorporated as a stable component of the adenovirus capsid. In addition, the nuclear assembly of adenoviruses may preclude correct post-translational modification of protein moieties that are normally processed through the secretory pathway. For these reasons, complex binding ligands, including antibodies, have so far only been successfully employed in twocomponent targeting strategies, where they were bound to the adenovirus fiber indirectly via a second protein moiety $[14,16,18,43-47,77]$. This leads to an increased target specificity by ablating CAR-mediated entry, since the viral fiber knob is blocked by the adaptor protein. A disadvantage of the bridging strategy employed by using bispecific adapter proteins in the retargeting of adenoviral vectors to tumor cells specifically lies in the fact that the targeting ligand has to be genetically incorporation into the adenoviral vector genome to avoid loss upon viral replication.

\section{GENOMIC ADAPTER TARGETED CRAd}

A single component targeted adenovirus was developed by van Beusechem et al. [78], who constructed a new type of targeted CRAd that carries an expression cassette for a secreted antibody targeting moiety in its genome. The targeting moiety was the bispecific single-chain [scFv] antibody 425 S11 consisting of the anti-epidermal growth factor receptor [EGFR] scFv 425 and anti-adenovirus fiber knob scFv S11 that targets adenovirus entry via EGFR $[47,79]$. In contrast to CAR, EGFR is commonly overexpressed in many tumor types [80]. The novel EGFR-targeted CRAd produced its own bispecific $\mathrm{scFv}$ targeting moiety, while replicating in CAR-deficient cancer cells, providing its progeny with CAR-independent infectivity. As a result, the EGFR-targeted CRAd exhibited an improved oncolytic potency on CARdeficient cancer cells, primary brain tumor specimens, and 3$\mathrm{D}$ tumor spheroids in vitro.

Carette et al. [24] constructed and characterized an oncolytic adenovirus, carrying mutated capsid proteins to abolish the promiscuous adenovirus native tropism and encoding the same EGFR-targeted bispecific adapter molecule. The new virus displayed a highly selective targeting profile, with reduced infection of EGFR-negative cells and efficient killing of EGFR-positive cancer cells including primary EGFRpositive osteosarcoma cells that are refractory to infection by 
conventional adenoviruses. Hence, this CRAd combines the therapeutic gains of targeting and oncolytic replication, in a stable single-component genetic anticancer medicine, with the flexibility of a two-component targeting strategy.

\section{DETARGETING ADENOVIRUS FROM NORMAL TISSUE}

Proper targeting of CRAds to tumor cells should fulfill two requirements; i.e., the virus should be engineered to transduce the cells of interest with high efficiency and its natural tropism towards nontargeted tissues should be abolished. To achieve strict targeting, the native tropism of the CRAd should be abolished. This is especially important for systemic delivery of CRAds because the vast majority of intravenously injected adenovirus is sequestered in the liver [12].

The presence of CAR on erythrocytes leads to a prolonged in vivo blood half-life and significantly reduced liver infection when a CAR-tropic Ad was injected intravenously [81]. In addition, integrins appear to mediate $\mathrm{Ad}$ binding to platelets. Platelets bound to Ad displaying an RGD ligand in the fiber knob more efficiently than unmodified Ad [82].

Recently, it was described that in addition to the CARintegrin pathway, blood-borne adenovirus infects hepatocytes through an indirect pathway that involves blood coagulation factors [83-85] which may bridge to cellular heparan sulfate glycosaminoglycans [HSG]. The HSG putative binding site KKTK of the Ad5 fiber shaft domain has been shown to be involved in Ad5 liver transduction in mice, rats and non-human primates [86]. Liu et al. [87] showed that preinjection of snake venom factor X-binding protein [X-bp] reduces hepatocyte transduction and increases the circulation time in blood of an intravenously injected, fiber-chimeric Ad5/35 vector. X-bp pretreatment resulted in improved Ad5/35 transduction of liver metastases and increased the antitumor efficacy of an Ad5/35-based oncolytic adenovirus.

Thus, detargeting adenovirus from normal tissue upon intravenous injection may require ablation of CAR, $\alpha \mathrm{v}$ integrin- or heparan sulfate glycosaminoglycans binding sites. Mutations that abolish CAR- and integrin-binding in the genome should produce a CRAd lacking CAR and $\alpha \mathrm{v}$ integrin-binding sites capable of producing targeted progeny upon replication in cancer cells [24]. Surprisingly, CAR and integrin double binding-ablated vectors had no effect on hepatocyte transduction in animal studies. However, shaft mutation that ablated HSG binding on the background of a normal capsid was sufficient to abrogate liver transduction in vivo [75].

\section{CONCLUSION}

In summary, conditionally replicating adenovirusses or oncolytic adenovirusses may be selectively targeted to cancer cells by the direct incorporation of specific peptides or by the expression of a selective adaptor molecule. The ultimate goal of these targeted viruses is to allow systemic administration to treat disseminated disease. Despite effective targeting and tumor eradication in vitro and in mouse models, the results from systemic administration of targeted CrAds in animal models are limited. In addition, clinical effects of CrAds are disappointing up till now. Therefore, combination therapies in which targeted CrAds are combined with radiation [88], chemotherapy $[89,90]$ or therapeutic genes such as TNF-related apoptosis-inducing ligand [TRAIL] [91, 92] or an antiangiogenic protein; vascular endothelial growth factor receptor [93] are investigated. These combination therapies are most likely to produce clinical effects.

$\begin{array}{lll}\text { ABBREVIATIONS } \\ \mathrm{CAR} & =\text { Coxsackie and adenovirus receptor } \\ \mathrm{EGFR} & = & \text { Epidermal growth factor receptor } \\ \mathrm{HIF} & = & \text { Hypoxia-inducible factor } \\ \mathrm{KDa} & = & \text { Kilo Dalton } \\ \mathrm{Rb} & = & \text { Retinoblastoma } \\ \mathrm{VEGF} & = & \text { Vascular epithelium growth factor } \\ \mathrm{CrAd} & = & \text { Conditionally replicating adenovirus } \\ \mathrm{RGD} & = & \text { Arginine-glycine-aspartic acid } \\ \mathrm{ScFV} & = & \text { Single-chain variable fragment } \\ \mathrm{TNF} & = & \text { The tumor necrosis factor }\end{array}$

\section{REFERENCES}

[1] Edelstein ML, Abedi MR, Wixon J. Gene therapy clinical trials worldwide to 2007-an update. J Gene Med 2007; 9(10): 83342.

[2] Alemany R, Balague C, Curiel DT. Replicative adenoviruses for cancer therapy. Nat Biotechnol 2000; 18(7): 723-7.

[3] Heise C, Kirn DH. Replication-selective adenoviruses as oncolytic agents. J Clin Investig 2000; 105(7): 847-51.

[4] Chroboczek J, Ruigrok RW, Cusack S. Adenovirus fiber 4. Curr Top Microbiol Immunol 1995; 199 (Pt 1):163-200.

[5] Sharma A, Li X, Bangari DS, Mittal SK. Adenovirus receptors and their implications in gene delivery 1. Virus Res 2009; 143(2): 18494.

[6] Liszewski MK, Post TW, Atkinson JP. Membrane cofactor protein [MCP or CD46]: newest member of the regulators of complement activation gene cluster 9. Annu Rev Immunol 1991; 9: 431-55.

[7] Bergelson JM, Cunningham JA, Droguett G, et al. Isolation of a common receptor for Coxsackie $B$ viruses and adenoviruses 2 and 5. Science 1997; 275(5304): 1320-3.

[8] Tomko RP, Xu R, Philipson L. HCAR and MCAR: the human and mouse cellular receptors for subgroup $\mathrm{C}$ adenoviruses and group B coxsackieviruses. Proc Natl Acad Sci USA 1997; 94(7): 3352-6.

[9] Bewley MC, Springer K, Zhang YB, et al. Structural analysis of the mechanism of adenovirus binding to its human cellular receptor, CAR 1. Science 1999; 286(5444): 1579-83.

[10] Wickham TJ, Mathias P, Cheresh DA, Nemerow GR. Integrins alpha $\mathrm{v}$ beta 3 and alpha $\mathrm{v}$ beta 5 promote adenovirus internalization but not virus attachment 1 . Cell 1993; 73(2): 309-19.

[11] Wang K, Huang S, Kapoor-Munshi A, Nemerow G. Adenovirus internalization and infection require dynamin. J Virol 1998; 72(4): 3455-8.

[12] Haisma HJ, Kamps JA, Kamps GK, et al. Polyinosinic acid enhances delivery of adenovirus vectors in vivo by preventing sequestration in liver macrophages 2. J Gen Virol 2008; 89(Pt 5): 1097105.

[13] Cripe TP, Dunphy EJ, Holub AD, et al. Fiber knob modifications overcome low, heterogeneous expression of the coxsackievirusadenovirus receptor that limits adenovirus gene transfer and onco- 
lysis for human rhabdomyosarcoma cells 1. Cancer Res 2001; 61(7): 2953-60.

[14] Grill J, Van B, V, Van D, V, et al. Combined targeting of adenoviruses to integrins and epidermal growth factor receptors increases gene transfer into primary glioma cells and spheroids. Clin Cancer Res 2001; 7(3): 641-50.

[15] Li Y, Pong RC, Bergelson JM, et al. Loss of adenoviral receptor expression in human bladder cancer cells: a potential impact on the efficacy of gene therapy 40. Cancer Res 1999; 59(2): 325-30.

[16] Miller CR, Buchsbaum DJ, Reynolds PN, et al. Differential susceptibility of primary and established human glioma cells to adenovirus infection: targeting via the epidermal growth factor receptor achieves fiber receptor-independent gene transfer 1. Cancer Res 1998; 58(24): 5738-48.

[17] Wesseling JG, Bosma PJ, Krasnykh V, et al. Improved gene transfer efficiency to primary and established human pancreatic carcinoma target cells via epidermal growth factor receptor and integrin-targeted adenoviral vectors 1 . Gene Ther 2001; 8(13): 96976.

[18] Witlox MA, Van B, V, Grill J, et al. Epidermal growth factor receptor targeting enhances adenoviral vector based suicide gene therapy of osteosarcoma 1. J Gene Med 2002; 4(5): 510-6.

[19] Okegawa T, Pong RC, Li Y, et al. The mechanism of the growthinhibitory effect of coxsackie and adenovirus receptor [CAR] on human bladder cancer: a functional analysis of car protein structure 1. Cancer Res 2001; 61(17): 6592-600.

[20] Zeimet AG, Muller-Holzner E, Schuler A, et al. Determination of molecules regulating gene delivery using adenoviral vectors in ovarian carcinomas 2. Gene Ther 2002; 9(16): 1093-100.

[21] Bischoff JR, Kirn DH, Williams A, et al. An adenovirus mutant that replicates selectively in p53-deficient human tumor cells 1 . Science 1996; 274(5286): 373-6.

[22] O'Shea CC, Johnson L, Bagus B, et al. Late viral RNA export, rather than p53 inactivation, determines ONYX-015 tumor selectivity 1. Cancer Cell 2004; 6(6): 611-23.

[23] Ganly I, Kirn D, Eckhardt G, et al. A phase I study of Onyx-015, an E1B attenuated adenovirus, administered intratumorally to patients with recurrent head and neck cancer 5. Clin Cancer Res 2000; 6(3): 798-806.

[24] Carette JE, Graat HC, Schagen FH, et al. A conditionally replicating adenovirus with strict selectivity in killing cells expressing epidermal growth factor receptor 2. Virology 2007; 361(1): 56-67.

[25] Nemunaitis J, Khuri F, Ganly I, et al. Phase II trial of intratumoral administration of ONYX-015, a replication-selective adenovirus, in patients with refractory head and neck cancer 2. J Clin Oncol 2001; 19(2): 289-98.

[26] Hecht JR, Bedford R, Abbruzzese JL, et al. A phase I/II trial of intratumoral endoscopic ultrasound injection of ONYX-015 with intravenous gemcitabine in unresectable pancreatic carcinoma 1. Clin Cancer Res 2003; 9(2): 555-61.

[27] Ries S, Korn WM. ONYX-015: mechanisms of action and clinical potential of a replication-selective adenovirus 2. Br J Cancer 2002; 86(1): 5-11.

[28] Kirn D. Clinical research results with d11520 [Onyx-015], a replication-selective adenovirus for the treatment of cancer: what have we learned? 7. Gene Ther 2001; 8(2): 89-98.

[29] Reid T, Galanis E, Abbruzzese J, et al. Hepatic arterial infusion of a replication-selective oncolytic adenovirus [d11520]: phase II viral, immunologic, and clinical endpoints 3. Cancer Res 2002; 62(21): 6070-9.

[30] Garber K. China approves world's first oncolytic virus therapy for cancer treatment 1. J Natl Cancer Inst 2006; 98(5): 298-300.

[31] Fueyo J, Gomez-Manzano C, Alemany R, et al. A mutant oncolytic adenovirus targeting the $\mathrm{Rb}$ pathway produces anti-glioma effect in vivo 1 . Oncogene 2000; 19(1): 2-12.

[32] Jiang H, Gomez-Manzano C, Lang FF, et al. Oncolytic adenovirus: preclinical and clinical studies in patients with human malignant gliomas 5. Curr Gene Ther 2009; 9(5): 422-7.

[33] Jiang H, Gomez-Manzano C, Lang FF, et al. Oncolytic adenovirus: preclinical and clinical studies in patients with human malignant gliomas 1. Curr Gene Ther 2009; 9(5):422-7.

[34] Chia MC, Shi W, Li JH, et al. A conditionally replicating adenovirus for nasopharyngeal carcinoma gene therapy 19. Mol Ther 2004; 9(6): 804-17.

[35] Balague C, Noya F, Alemany R, et al. Human papillomavirus E6E7-mediated adenovirus cell killing: selectivity of mutant adenovirus replication in organotypic cultures of human keratinocytes 11. J Virol 2001; 75(16): 7602-11.

[36] Cattaneo R, Miest T, Shashkova EV, Barry MA. Reprogrammed viruses as cancer therapeutics: targeted, armed and shielded 12 . Nat Rev Microbiol 2008; 6(7): 529-40.

[37] Nettelbeck DM, Rivera AA, et al. Novel oncolytic adenoviruses targeted to melanoma: Specific viral replication and cytolysis by expression of E1A mutants from the tyrosinase enhancer/promoter 8. Cancer Res 2002; 62(16): 4663-70.

[38] Rocconi RP, Zhu ZB, Stoff-Khalili M, et al. Treatment of ovarian cancer with a novel dual targeted conditionally replicative adenovirus [CRAd] 10. Gynecologic Oncol 2007; 105(1):113-21.

[39] Deweese TL, van der PH, Li S, et al. A phase I trial of CV706, a replication-competent, PSA selective oncolytic adenovirus, for the treatment of locally recurrent prostate cancer following radiation therapy 3. Cancer Res 2001; 61(20): 7464-72.

[40] Rocconi RP, Zhu ZB, Stoff-Khalili M, et al. Treatment of ovarian cancer with a novel dual targeted conditionally replicative adenovirus [CRAd] 10. Gynecologic Oncol 2007; 105(1):113-21.

[41] Saukkonen K, Hemminki A. Tissue-specific promoters for cancer gene therapy 17. Expert Opin Biol Ther 2004; 4(5): 683-96.

[42] Krasnykh VN, Douglas JT, Van B, V. Genetic targeting of adenoviral vectors. Mol Ther 2000; 1(5 Pt 1): 391-405.

[43] Dmitriev I, Kashentseva E, Rogers BE, et al. Ectodomain of coxsackievirus and adenovirus receptor genetically fused to epidermal growth factor mediates adenovirus targeting to epidermal growth factor receptor-positive cells 1 . J Virol 2000; 74(15): 6875-84.

[44] Douglas JT, Rogers BE, Rosenfeld ME, et al. Targeted gene delivery by tropism-modified adenoviral vectors 3 . Nat Biotechnol 1996; 14(11):1574-8.

[45] Doukas J, Hoganson DK, Ong M, et al. Retargeted delivery of adenoviral vectors through fibroblast growth factor receptors involves unique cellular pathways 1. FASEB J 1999; 13(11): 145966.

[46] Haisma HJ, Pinedo HM, Rijswijk A, et al. Tumor-specific gene transfer via an adenoviral vector targeted to the pan-carcinoma antigen EpCAM 20. Gene Ther 1999; 6(8): 1469-74.

[47] Haisma HJ, Grill J, Curiel DT, et al. Targeting of adenoviral vectors through a bispecific single-chain antibody. Cancer Gene Ther 2000; 7(6): 901-4.

[48] Romanczuk H, Galer CE, Zabner J, et al. Modification of an adenoviral vector with biologically selected peptides: a novel strategy for gene delivery to cells of choice 1. Hum Gene Ther 1999; 10(16): 2615-26.

[49] Haviv YS, Blackwell JL, Kanerva A, et al. Adenoviral gene therapy for renal cancer requires retargeting to alternative cellular receptors 3. Cancer Res 2002; 62(15): 4273-81.

[50] Zhu ZB, Mathis JM, Makhija SK, et al. Targeting of a conditionally replicative adenovirus agent to human squamous cell carcinomas of the head and neck 1. Int J Oncol 2007; 31(5): 1213-22.

[51] Ramirez PJ, Vickers SM, Ono HA, et al. Optimization of conditionally replicative adenovirus for pancreatic cancer and its evaluation in an orthotopic murine xenograft model 2. Am J Surg 2008; 195(4): 481-90.

[52] Ramirez PJ, Vickers SM, Ono HA, et al. Optimization of conditionally replicative adenovirus for pancreatic cancer and its evaluation in an orthotopic murine xenograft model 2. Am J Surg 2008; 195(4): 481-90.

[53] Stoff-Khalili MA, Rivera AA, Stoff A, et al. Combining high selectivity of replication via CXCR4 promoter with fiber chimerism for effective adenoviral oncolysis in breast cancer 3 . Int $\mathbf{J}$ Cancer 2007; 120(4): 935-41.

[54] Guse K, Diaconu I, Rajecki M, et al. Ad5/3-9HIF-Delta24VEGFR-1-Ig, an infectivity enhanced, dual-targeted and antiangiogenic oncolytic adenovirus for kidney cancer treatment 3. Gene Ther 2009; 16(8): 1009-20.

[55] Hoffmann D, Meyer B, Wildner O. Improved glioblastoma treatment with Ad5/35 fiber chimeric conditionally replicating adenoviruses 2. J Gene Med 2007; 9(9): 764-78.

[56] Suzuki K, Fueyo J, Krasnykh V, et al. A conditionally replicative adenovirus with enhanced infectivity shows improved oncolytic potency 1. Clin Cancer Res 2001; 7(1): 120-6.

[57] Shinoura N, Yoshida Y, Tsunoda R, et al. Highly augmented cytopathic effect of a fiber-mutant E1B-defective adenovirus for gene therapy of gliomas. Cancer Res 1999; 59(14): 3411-6. 
[58] Bauerschmitz GJ, Lam JT, Kanerva A, et al. Treatment of ovarian cancer with a tropism modified oncolytic adenovirus 14. Cancer Res 2002; 62(5): 1266-70.

[59] Chu RL, Post DE, Khuri FR, Van Meir EG. Use of replicating oncolytic adenoviruses in combination therapy for cancer 2. Clin Cancer Res 2004; 10(16): 5299-312.

[60] Khuri FR, Nemunaitis J, Ganly I, et al. A controlled trial of intratumoral ONYX-015, a selectively-replicating adenovirus, in combination with cisplatin and 5-fluorouracil in patients with recurrent head and neck cancer 2. Nat Med 2000; 6(8): 879-85.

[61] Jiang H, Gomez-Manzano C, Aoki H, et al. Examination of the therapeutic potential of Delta-24-RGD in brain tumor stem cells: role of autophagic cell death 16. J Natl Cancer Inst 2007; 99(18):1410-4.

[62] Jiang H, Gomez-Manzano C, Aoki H, et al. Examination of the therapeutic potential of Delta-24-RGD in brain tumor stem cells: role of autophagic cell death 16. J Natl Cancer Inst 2007; 99(18): $1410-4$.

[63] Lamfers MLM, Grill J, Dirven CMF, et al. Potential of the conditionally replicative adenovirus Ad5-Delta 24RGD in the treatment of malignant gliomas and its enhanced effect with radiotherapy 7 . Cancer Res 2002; 62(20): 5736-42.

[64] Cascallo M, Alonso MM, Rojas JJ, et al. Systemic toxicity-efficacy profile of ICOVIR-5, a potent and selective oncolytic adenovirus based on the pRB pathway 2. Mol Ther 2007; 15(9):1607-15.

[65] Cascallo M, Alonso MM, Rojas JJ, et al. Systemic toxicity-efficacy profile of ICOVIR-5, a potent and selective oncolytic adenovirus based on the pRB pathway 2. Mol Ther 2007; 15(9): 1607-15.

[66] Ranki T, Kanerva A, Ristimaki A, et al. A heparan sulfate-targeted conditionally replicative adenovirus, Ad5.pk7-Delta24, for the treatment of advanced breast cancer 3. Gene Ther 2007; 14(1): 5867.

[67] Piao Y, Jiang H, Alemany R, et al. Oncolytic adenovirus retargeted to Delta-EGFR induces selective antiglioma activity 1. Cancer Gene Ther 2009; 16(3): 256-65.

[68] Ulasov IV, Zhu ZB, Tyler MA, et al. Survivin-driven and fibermodified oncolytic adenovirus exhibits potent antitumor activity in established intracranial glioma 25. Hum Gene Ther 2007; 18(7): 589-602.

[69] Nishimoto T, Yoshida K, Miura Y, et al. Oncolytic virus therapy for pancreatic cancer using the adenovirus library displaying random peptides on the fiber knob 1 . Gene Ther 2009; 16(5): 66980 .

[70] Belousova N, Krendelchtchikova V, Curiel DT, Krasnykh V. Modulation of adenovirus vector tropism via incorporation of polypeptide ligands into the fiber protein 1. J Virol 2002; 76(17):862131.

[71] Wickham TJ, Tzeng E, Shears LL, et al. Increased in vitro and in vivo gene transfer by adenovirus vectors containing chimeric fiber proteins 4. J Virol 1997; 71(11): 8221-9.

[72] Yoshida Y, Sadata A, Zhang W, et al. Generation of fiber-mutant recombinant adenoviruses for gene therapy of malignant glioma. Hum Gene Ther 1998; 9(17): 2503-15.

[73] Parks RJ. Adenovirus protein IX: a new look at an old protein 1. Mol Ther 2005; 11(1):19-25.

[74] Meulenbroek RA, Sargent KL, Lunde J, et al. Use of adenovirus protein IX [pIX] to display large polypeptides on the virion-generation of fluorescent virus through the incorporation of pIXGFP 2. Mol Ther 2004; 9(4): 617-24.

[75] Bayo-Puxan N, Gimenez-Alejandre M, et al. Replacement of adenovirus type 5 fiber shaft heparan sulfate proteoglycan-binding domain with RGD for improved tumor infectivity and targeting 1 . Hum Gene Ther 2009; 20(10): 1214-21.
[76] Vigne E, Mahfouz I, Dedieu JF, Brie A, et al. RGD inclusion in the hexon monomer provides adenovirus type 5-based vectors with a fiber knob-independent pathway for infection. J Virol 1999; 73(6): 5156-61.

[77] Nettelbeck DM, Rivera AA, Volk AL, et al. Engineering of oncolytic adenoviruses for targeted and efficient virotherapy of malignant melanoma 4. Mol Ther 2003; 7(5): S355-S356.

[78] Van B, V, Mastenbroek DC, van den Doel PB, et al. Conditionally replicative adenovirus expressing a targeting adapter molecule exhibits enhanced oncolytic potency on CAR-deficient tumors. Gene Ther 2003;10(23): 1982-91.

[79] Van B, V, Grill J, Mastenbroek DC, et al. Efficient and selective gene transfer into primary human brain tumors by using singlechain antibody-targeted adenoviral vectors with native tropism abolished. J Virol 2002; 76(6): 2753-62.

[80] Kolibaba KS, Druker BJ. Protein tyrosine kinases and cancer 2. Biochim Biophys Acta 1997; 13339(3):F217-F248.

[81] Seiradake E, Henaff D, Wodrich $\mathrm{H}$, et al. The cell adhesion molecule "CAR" and sialic acid on human erythrocytes influence adenovirus in vivo biodistribution 1. PLoS Pathog 2009; 5(1): e1000277.

[82] Shimony N, Elkin G, Kolodkin-Gal D, et al. Analysis of adenoviral attachment to human platelets 1. Virol J 2009; 6: 25 .

[83] Parker AL, Waddington SN, Nicol CG, et al. Multiple vitamin Kdependent coagulation zymogens promote adenovirus-mediated gene delivery to hepatocytes 3. Blood 2006; 108(8): 2554-61.

[84] Waddington SN, McVey JH, Bhella D, et al. Adenovirus serotype 5 hexon mediates liver gene transfer 5 . Cell 2008; 132(3): 397-409.

[85] Shayakhmetov DM, Gaggar A, Ni S, Li ZY, Lieber A. Adenovirus binding to blood factors results in liver cell infection and hepatotoxicity 10. J Virol 2005; 79(12): 7478-91.

[86] Gimenez-Alejandre M, Cascallo M, Bayo-Puxan N, Alemany R. Coagulation factors determine tumor transduction in vivo 2. Hum Gene Ther 2008; 19(12): 1415-9.

[87] Liu Y, Wang HJ, Yumul R, et al. Transduction of Liver Metastases After Intravenous Injection of Ad5/35 or Ad35 Vectors With and Without Factor X-Binding Protein Pretreatment 3. Hum Gene Ther 2009; 20(6): 621-9.

[88] Lamfers MLM, Grill J, Dirven CMF, et al. Potential of the conditionally replicative adenovirus Ad5-Delta 24RGD in the treatment of malignant gliomas and its enhanced effect with radiotherapy 7 . Cancer Res 2002; 62(20):5736-42.

[89] Khuri FR, Nemunaitis J, Ganly I, et al. A controlled trial of intratumoral ONYX-015, a selectively-replicating adenovirus, in combination with cisplatin and 5-fluorouracil in patients with recurrent head and neck cancer 2. Nat Med 2000; 6(8): 879-85.

[90] Nemunaitis J, Swisher SG, Timmons T, et al. Adenovirusmediated p53 gene transfer in sequence with cisplatin to tumors of patients with non-small-cell lung cancer 4. J Clin Oncol 2000; 18(3): 609-22.

[91] Sova P, Ren XW, Ni S, et al. A tumor-targeted and conditionally replicating oncolytic adenovirus vector expressing TRAIL for treatment of liver metastases 1. Mol Ther 2004; 9(4): 496-509.

[92] Bremer E, van Dam GM, de Bruyn M, et al. Potent Systemic Anticancer Activity of Adenovirally Expressed EGFR-Selective TRAIL Fusion Protein 69. Mol Ther 2008; 16(12):1919-26.

[93] Guse K, Diaconu I, Rajecki M, et al. Ad5/3-9HIF-Delta24VEGFR-1-Ig, an infectivity enhanced, dual-targeted and antiangiogenic oncolytic adenovirus for kidney cancer treatment 4 . Gene Ther 2009; 16(8):1009-20

(C) Haisma and Geerts; Licensee Bentham Open.

This is an open access article licensed under the terms of the Creative Commons Attribution Non-Commercial License (http://creativecommons.org/licenses/by-nc/3.0/) which permits unrestricted, non-commercial use, distribution and reproduction in any medium, provided the work is properly cited. 